\title{
LE TOURNANT MAL NÉGOCIÉ DE LA RECONSTRUCTION AGRICOLE EN FRANCE APRÈS LA PREMIËRE GUERRE MONDIALE (1920-1939)
}

\author{
Thierry Pouch ${ }^{1}$
}

Université de Reims Champagne Ardenne, Chambres d'agriculture France à Paris (APCA)

Au lendemain de la signature du Traité de Versailles, la question de la disponibilité en biens alimentaires se pose en France avec une certaine acuité. L'économie française est en effet sortie très affectée par le conflit militaire mondial et les pouvoirs publics s'interrogent sur la capacité de l'agriculture nationale à répondre aux besoins alimentaires d'une population dont une fraction, majoritairement paysanne, a été envoyée sur le front. De plus, le territoire national a été amputé de plusieurs millions d'hectares de terre cultivables durant le conflit. Outre ce questionnement sur la capacité à produire des denrées agricoles et alimentaires, c'est la vision du devenir du secteur agricole comme levier du redressement national, qui suppose un effort de modernisation des structures de production, qui fait débat entre les deux guerres mondiales. L'article revient sur cette double dimension et montre que les gouvernements successifs, jusqu'à l'avènement du Front populaire, ont échoué à instaurer une dynamique de modernisation de l'agriculture française, faute d'avoir défini une politique agricole active.

Mots clés : agriculture française, politique agricole, histoire économique, analyse des crises agricoles.

1. L'auteur remercie les deux rapporteurs anonymes ainsi que Marine Raffray (APCA, Service études) pour leurs remarques et suggestions, qui ont permis d'améliorer une version antérieure de cet article. Il reste bien évidement seul responsable des erreurs et omissions qui subsisteraient. 
«Une Europe improductive, inoccupée, désorganisée, déchirée par des querelles intestines et les haines internationales, est devant nous. Elle lutte, elle meurt de faim, elle pille, elle ment. Comment pourrionsnous la peindre sous de moins sombres couleurs? »

John Maynard Keynes

Les conséquences économiques de la paix - 1919

ris dans son sens premier, le Traité de Versailles signé le 28 juin 1919 et promulgué le 10 janvier 1920, est un Traité instaurant la paix entre les belligérants. Qu'il ait été l'objet de nombreuses critiques - en particulier, du côté des hommes politiques, celles émanant de l'Allemagne vaincue et, concernant les économistes, celles, incisives, virulentes autant qu'anticipatrices, exprimées par John Maynard Keynes dans son célèbre ouvrage daté de 1919, Les conséquences économiques de la paix - ne remet pas en question le fait que les nations qui sont entrées en guerre six ans auparavant se sont fixé une ambition : celle de reconstruire leurs outils de production, leurs infrastructures, et donc de préparer l'avenir des générations futures après le traumatisme de la guerre. La France au premier chef entendait restaurer son rang sur l'échiquier économique international, en s'appuyant notamment sur son secteur agricole. Car, comme le rappelait J.-M. Keynes dans sa préface à l'édition française de son livre de 1919, si la situation politique et morale de la France ne souffrait après la victoire, d'aucune ambiguiité, l'état de son économie était plutôt déplorable, au point disait-il de compromettre son avenir. Le secteur agricole de la France, mal remis d'une grave crise à la fin du siècle précédent, était en effet sorti exsangue de la Première Guerre mondiale.

Or, s'agissant d'un secteur économique aussi important à l'époque au regard de son poids dans le Produit intérieur brut et dans la population active, la grande priorité de l'État français dès l'Armistice signé en novembre 1918 et la fin des hostilités, fut de rétablir les conditions de la production agricole afin de desserrer la contrainte d'approvisionnement alimentaire qui avait pesé si fortement durant toute la durée du conflit. Par extension, c'est précisément parce qu'elle symbolise une certaine idée de la Nation, entretient un lien affectif et historique avec le peuple français, constitue en quelque sorte l'épine dorsale de la société française, que l'agriculture, au-delà de sa capacité à nourrir le pays, était appréhendée comme l'un des vecteurs efficaces du redressement de la France. De ce point de vue, toutes les analyses historiques semblent converger pour dire que ce renouveau économique et moral 
de la France passait nécessairement par une agriculture réformée, renforcée, c'est-à-dire accomplissant le virage de la modernité.

Un siècle après un Traité de Versailles instituant une paix aussi précaire que courte, se pencher sur la reconstruction de l'agriculture française suggère de montrer en quoi ce secteur avait besoin d'un soutien politique et économique puissant et massif pour non seulement résoudre la question de l'insécurité alimentaire qui caractérisait le pays, mais pour en faire un levier du redressement national. Ce qu'il est important de montrer, c'est que les initiatives prises par l'État français n'ont pas permis aux acteurs du monde agricole d'opérer ce tournant de la modernité. En revanche, les actions menées à partir du milieu de la décennie 1930, par le Front populaire, ont formé quant à elles les signes annonciateurs d'une politique agricole qui, une quinzaine d'années après les Traité de Versailles, préfigure le saut dans la modernité économique de l'agriculture française, ou, pour user d'un terme qui fera florès par la suite pour finalement subir un lourd discrédit, dans le « productivisme».

L'objet de cet article est de montrer en quoi le processus de paix n'a pas véritablement constitué un levier suffisant pour que l'agriculture française sorte de l'ornière dans laquelle elle se trouvait depuis le dernier tiers du XIX ${ }^{e}$ siècle. Au gré des pesanteurs politiques, des priorités des gouvernements, des divergences au sein même du monde agricole, la trajectoire de la modernisation des outils de production s'inscrit en réalité sur un temps bien plus long. La France entre en guerre avec un handicap agricole, puisqu'elle porte encore les stigmates de la crise agricole du XIXe siècle et le conflit armé a entravé le difficile processus de modernisation de l'agriculture. C'est pourquoi la première partie de cet article fera un retour sur la situation économique de l'agriculture française avant et pendant les hostilités. L'avènement de la paix, dont le Traité de Versailles constitue le point culminant, oblige le politique et les économistes à prendre des décisions dont la finalité est de redresser la production agricole, de s'affranchir des importations massives pratiquées entre 1914 et 1918, voire même après. La deuxième partie de l'article traitera de ces décisions et montrera en quoi elles ont été insuffisantes pour rétablir la production et placer le secteur agricole sur la voie de la modernité. Enfin, la dernière partie de l'article montrera en quoi la Grande Dépression des années 1930 plonge une fois de plus l'agriculture dans la crise. Les dispositions prises par le Front populaire en matière agricole dessinent ce qui deviendra après la guerre la politique agricole moderne. 


\section{Retard de l'agriculture française ou pas : I'interminable controverse}

Il y aurait comme un « invariant structurel » qui distingue l'histoire de l'agriculture française, qui la traverse de décennie en décennie, voire de siècle en siècle. Cet invariant, qui oppose les économistes, c'est celui qui porte sur la capacité de la France à se nourrir elle-même, à s'auto suffire, et, à certaines époques, à espérer exporter, afin de faire du pays une puissance qui compte sur les marchés internationaux. Encore aujourd'hui, la problématique de la sécurité et de l'autosuffisance alimentaires, ponctuent les débats au sein d'une société bousculée par une pandémie. L'entrée en guerre puis la phase de la reconstruction du secteur agricole forment des moments emblématiques d'une controverse qui, à bien y regarder, s'étend de la fin du $\mathrm{XIX}^{\mathrm{e}}$ siècle à aujourd'hui. À la moindre fluctuation conjoncturelle des productions agricoles, à la moindre crise, les débats repartent de plus belle.

\subsection{La crise agricole du dernier tiers du $X I X X^{e}$ siècle}

L'entrée en guerre de la France, comme des autres belligérants, va rapidement poser la question de l'approvisionnement alimentaire de la population, c'est-à-dire des familles et des soldats partis au front. Le défi lancé aux autorités gouvernementales est colossal, complexe, dans la mesure où l'agriculture française sort à peine d'une crise qui, selon Michel Augé-Laribé (1950), s'étale de 1878 à 1914. Plus de trente ans de crise pour le secteur agricole sur laquelle il faut brièvement revenir. En réalité, une crise un peu moins longue, car le redressement de la production au tout début du $X X^{e}$ siècle ramène sa durée à une vingtaine d'années. Même statistiquement et économiquement raccourcie, la crise agricole est profonde (Zolla, 1904 ; Augé-Laribé, 1950). Elle se distingue par une baisse des prix, de gros comme de détail, les premiers passant d'un pic atteint en 1877 à un plancher vingt ans après (indice 140 à 88, selon I'Annuaire statistique de la France, 1961), les seconds étant également emportés dans un mouvement baissier important, comme le vin, le blé, et, dans une moindre mesure, la pomme de terre. L'analyse des faits économiques indique que ces baisses de prix agricoles s'inscrivent dans un cycle Kondratieff descendant, qui s'étale sur presque un quart de siècle, de 1873 à 1896.

L'agriculture est donc prise dans une tendance baissière généralisée des prix (Lhomme, 1970). L'interprétation économique de ces baisses de prix agricoles conduit à privilégier deux facteurs déterminants. Le 
premier, décisif pour les années qui vont suivre, est le retard technique accumulé par les agriculteurs français, en particulier dans le domaine de l'usage des intrants chimiques et des machines. Michel Augé-Laribé pointe la responsabilité des pouvoirs publics pour ce qui est de l'organisation des conditions de diffusion de ces progrès techniques notamment pour les cultures, mais aussi, et surtout, celle des agriculteurs, lesquels sont non seulement dépourvus de moyens financiers, mais restent enfermés dans des schémas de production périmés, hérités des générations antérieures de paysans (Augé-Laribé, op. cit.). La crise agricole est selon lui une crise d'adaptation, la manifestation d'un refus, ou d'une réticence des agriculteurs à prendre le tournant du progrès. À l'orée du XXe siècle, et à quelques encablures de la guerre, la production s'accroît mais les rendements demeurent faibles, sans commune mesure avec ceux réalisés par les concurrents européens de la France (tableau 1).

Tableau 1. L'évolution des rendements du blé

Quintaux/habitants

\begin{tabular}{lrrr} 
& 1880 & 1890 & 1900 \\
Belgique & 15,6 & - & 22,5 \\
Allemagne & 12,9 & 14,4 & 18,7 \\
Angleterre & 16,4 & 20,6 & 19,2 \\
Hollande & 16,9 & 17,7 & 20,4 \\
Danemark & 24,6 & 22,2 & 27,5 \\
France & 11,0 & 12,7 & 12,9 \\
\hline
\end{tabular}

Source : Annuaire statistique de la France, année 1938.

Le second facteur explicatif du retard français en matière agricole réside dans la montée de la concurrence internationale, largement facilitée par le développement des moyens de transport, qu'ils soient ferroviaires ou maritimes, l'ouverture du Canal de Suez en 1869 ayant dans ce dernier cas joué un rôle éminent par la réduction des coûts de transport qu'il a occasionnée. Céréales canadiennes, américaines - le blé étant en pointe sur ce secteur -, laine en provenance d'Australie, viande bovine exportée par les Argentins, quand ce n'est pas au sein même des colonies que la menace se niche, à l'instar des productions viticoles algériennes qui concurrencent celles de la métropole..., des produits issus de l'agriculture qui, en plus de leurs coûts de production inférieurs à ceux des agriculteurs français, bénéficient des progrès réalisés dans le domaine des transports de marchandises, accentuant 
ainsi la pression concurrentielle sur les productions hexagonales. Sur la période 1880-1900, le solde de la balance commerciale agricole est constamment déficitaire. Un répit s'enclenche à partir de 1892, lors de I'instauration des tarifs douaniers Méline, permettant de réduire ce déficit par le truchement d'un protectionnisme défensif.

Brièvement rappelées, les performances, toutes relatives, de l'agriculture française à la veille de la guerre, nourrissent les clivages entre les économistes et entre les politiques. Les voix discordantes se font entendre, les optimistes, plutôt situés du côté des politiques ou bien des acteurs professionnels, estimant que la croissance des productions agricoles de la France jusqu'en 1900 la protège des pénuries, les autres, surtout des économistes, mettant en exergue le retard du secteur en le comparant à ses principaux concurrents, qui hypothèque la nécessaire couverture des besoins alimentaires du pays.

\subsection{La guerre et la question de l'approvisionnement alimentaire de la France}

L'accumulation des tensions géopolitiques entre les nations débouche en août 1914 sur une guerre dont beaucoup pensent qu'elle sera courte. Cette considération a son importance pour l'agriculture nationale. Sur les quelque 5,2 millions d'actifs agricoles que compte le pays à la veille de la guerre, près de 2 millions vont être mobilisés pour rejoindre le front, le chiffre de 3 millions ayant été atteint en 1918, ce qui représente près de $60 \%$ des agriculteurs. La période estivale, celle des moissons, n'est guère propice à l'envoi de paysans sur les champs de bataille. Qu'importe, la guerre étant appelée à ne pas se prolonger au-delà de trois mois, ces paysans seront de retour dans leurs fermes. Funeste erreur d'appréciation, qui se traduit par une certaine inertie des pouvoirs publics en matière d'actions à mener pour adapter les structures et la production à cette nouvelle donne. Sur la durée, la capacité du pays à s'auto approvisionner, à l'abri de surcroît des importations par les tarifs Méline, vole en éclat. Dès 1915, et jusqu'aux derniers combats livrés, la France recourt massivement aux importations de produits agricoles et alimentaires en provenance de l'étranger. À l'issue de la guerre, le déficit commercial agroalimentaire du pays représente plus de $35 \%$ du déficit global de l'économie française (Gervais, Jollivet, Tavernier, 1977).

L'invasion de nombreux départements par l'armée allemande (Somme, Oise, Marne, Aisne, Meuse, Vosges, Ardennes...) ampute le 
pays de plusieurs milliers d'hectares, soustrayant à l'outil de production de quoi produire pour nourrir la population civile, mais aussi les soldats sur le front (pain et vin en particulier). Elle s'ajoute aux dégradations des sols, occasionnées par les combats, à la réquisition des animaux, en particulier les équins, et du transport ferroviaire, et, surtout, à la pénurie d'hommes, qu'ils soient agriculteurs ou bien maréchaux ferrants, forgerons.... Ont été dénombrés, à la fin des hostilités, les paysans tués aux combats, près de 600000 sur les plus de 3 millions mobilisés, pour prendre la mesure des difficultés du secteur agricole à restaurer les conditions de la production (Charle, 2001).

C'est pourquoi l'entrée en guerre provoque un changement de régime politique et économique. À l'ordre libéral qui s'étend jusqu'au début du $X X^{e}$ siècle, se substitue un régime économique interventionniste assorti de la célèbre "Union sacrée ", pour à la fois gérer un conflit - dont les répercussions sur les approvisionnements en produits agricoles et alimentaires vont être nombreuses, et auxquelles s'ajoutent les contraintes pesant sur les disponibilités en armement et en munitions notamment - et préparer la reconstruction d'après-guerre (Kuisel, 1984 ; Anizan, 2014 ; Bonin, 2016). Les agriculteurs au front, il s'agit de garantir coûte que coûte les cultures, la production de denrées alimentaires. Cette ambition passe par la mobilisation des femmes, lesquelles sont appelées, par la voix de René Viviani, Président du Conseil au début de la Guerre, à ensemencer les champs, à récolter les denrées, quitte à établir des correspondances épistolaires avec leurs époux partis au front, afin de recueillir des conseils pour mettre en culture et récolter (voir son célèbre discours du 8 août 1914, «Debout, donc, les femmes françaises, jeunes enfants, filles et fils de la Patrie! Remplacez sur le champ de travail ceux qui sont sur le champ de bataille »). Ce sont près de 800000 exploitations agricoles qui, en France, durant le conflit, sont pilotées par des femmes (Vigreux, 2006).

Pour surmonter la désorganisation des campagnes, le gouvernement fait aussi appel aux personnes âgées et aux enfants, ainsi qu'à près de 150000 travailleurs issus des colonies ${ }^{2}$. L'interventionnisme étatique durant la guerre suscite quelques réactions hostiles, suspicieuses, dont celle de Michel Augé-Laribé, lequel, dans son ouvrage de

2. C'est le 20 mars 1915 qu'est ainsi créée la Commission de la main-d'œuvre agricole, dont la mission est justement de coordonner les initiatives pour faciliter les recrutements de salariés agricoles, qu'ils proviennent des colonies, ou bien encore d'Espagne ou du Portugal, deux pays qui se sont tenus à l'écart des hostilités. 
1950 dédié à la politique agricole de la France entre 1880 et 1940, indique que « le socialisme d'État entre dans l'expérience quotidienne, non pour la paix, mais pour la guerre. Tout va être administré » (page 305). Le point culminant de la politique menée par le gouvernement en matière d'approvisionnement de la population, des soldats mais aussi du bétail, est atteint avec le recours massif aux importations de céréales en provenance des gtats-Unis et du Canada. Car malgré les dispositifs politiques retenus et appliqués, les récoltes ne sont pas à la hauteur. Augé-Laribé parle même de dégringolade, constat cohérent avec la réduction des surfaces cultivables, la pénurie de main-d'œuvre, les réquisitions d'animaux, qui précipitent les productions végétales et animales dans la chute, compromettant ainsi les objectifs gouvernementaux (tableau 2).

Tableau 2. L'évolution des récoltes en France

Indice 100

\begin{tabular}{lrrrrrrr} 
& 1913 & 1914 & 1915 & 1916 & 1917 & 1918 & 1919 \\
Blé & 98 & 87 & 68 & 63 & 41 & 69 & 56 \\
Méteil & 88 & 76 & 65 & 63 & 47 & 53 & 53 \\
Seigle & 97 & 84 & 64 & 64 & 47 & 55 & 55 \\
Orge & 107 & 100 & 71 & 85 & 85 & 61 & 51 \\
Sarrasin & 122 & 115 & 100 & 58 & 82 & 48 & 58 \\
Avoine & 107 & 95 & 71 & 83 & 64 & 53 & 51 \\
Maïs & 96 & 102 & 77 & 75 & 68 & 45 & 45 \\
\hline
\end{tabular}

Source: Augé-Laribé, La politique agricole de la France (1880-1940), page 325.

Une telle évolution va porter un lourd discrédit à la politique douanière menée précédemment par Jules Méline, dont la finalité avait été de protéger les agriculteurs français de la concurrence de nouveaux producteurs, et par voie de conséquence, de garantir la sécurité alimentaire de la nation, y compris en cas de conflit militaire. C'était sans compter sur la durée de la guerre, mais également sur l'occupation d'une partie du territoire par l'adversaire allemand. On voit ainsi que, avant comme pendant la guerre, l'action politique produit des controverses entre les économistes, notamment au sujet du protectionnisme. Selon Augé-Laribé, le protectionnisme n'est pas adapté en temps de guerre. Pour faire face aux baisses drastiques des productions, à une couverture des besoins alimentaires insuffisante et aux risques de pénuries, le gouvernement décide dès le mois d'août 1914, par décrets successifs, de suspendre les droits de douane - ceux 
instaurés en 1892 par Jules Méline et qui furent consolidés en 1910, juste avant la guerre - sur un certain nombre de produits (froment, épeautre, farines, orge, avoine, légumes secs et viandes réfrigérées, pommes de terre, maïs en grains...) de moins en moins disponibles, et pour contenir l'inflation sur les prix alimentaires. Parallèlement, il s'agit de retenir une production affaiblie sur le territoire, en actionnant des dispositifs prohibant les exportations, et en procédant à des réquisitions, notamment de céréales. Un État qui décide donc d'agir, en fonction de l'état de la conjoncture agricole, de l'approvisionnement de la population et des troupes, tout en cherchant à établir un équilibre forcément fragile entre les agriculteurs restés dans les fermes, et les consommateurs qui ont à se nourrir. Dit autrement, ne pas mécontenter les acteurs du monde social.

Il en découle que pour nourrir un pays en guerre, le recours aux importations est inévitable. Les achats de céréales constituent, aux premiers jours des hostilités, le premier poste des importations, illustrant l'anticipation du gouvernement pour approvisionner le pays avec une certaine régularité. À moins, selon certains historiens, que cette ouverture des frontières aux importations ne soit en réalité qu'une réponse improvisée face à l'urgence. Il faudra attendre 1922, le temps que se reconstitue la production céréalière nationale, pour que les importations reviennent à leur niveau d'avant-guerre (tableau 3). Ces céréales, d'où proviennent-elles ? Essentiellement des États-Unis, puissance agricole qui s'affirme depuis le milieu du XIX ${ }^{e}$ siècle, et qui, entrant tardivement dans la guerre, détient des ressources agricoles importantes, indissociables de ses avancées techniques et agronomiques. Ils sont suivis de l'Argentine, de l'Australie et du Canada. Pour les producteurs américains, exporter vers l'Europe et singulièrement vers la France, constitue une opportunité économique, qui les conduit

Tableau 3. Parts des produits importés par la France

En \% du total des importations en valeur

\begin{tabular}{lrrrrrrrrr} 
& 1914 & 1915 & 1916 & 1917 & 1918 & 1919 & 1920 & 1921 & 1922 \\
Coton & 5,20 & 3,43 & 3,24 & 4,84 & 5,16 & 4,20 & 6,12 & 4,99 & 7,48 \\
Laines & 8,09 & 1,90 & 1,78 & 1,51 & 1,28 & 5,24 & 5,73 & 4,43 & 7,25 \\
Céréales & 10,39 & 9,97 & 9,55 & 8,61 & 9,21 & 7,70 & 9,50 & 7,05 & 3,82 \\
Viandes & 0,97 & 4,09 & 2,81 & 2,32 & 3,60 & 3,80 & 1,35 & 1,03 & 0,99 \\
Vins & 3,45 & 3,11 & 2,24 & 2,73 & 2,01 & 2,02 & 1,15 & 2,24 & 3,52 \\
\hline
\end{tabular}

Source : S. Becuwe et B. Blancheton (2017). 
à accumuler des revenus en hausse jusqu'à la fin de la guerre, assurant ainsi leur prospérité dans les années précédant la Grande Dépression (graphique 1) (Pouch, 2014).

\section{Graphique 1. Revenu agricole net des États-Unis}

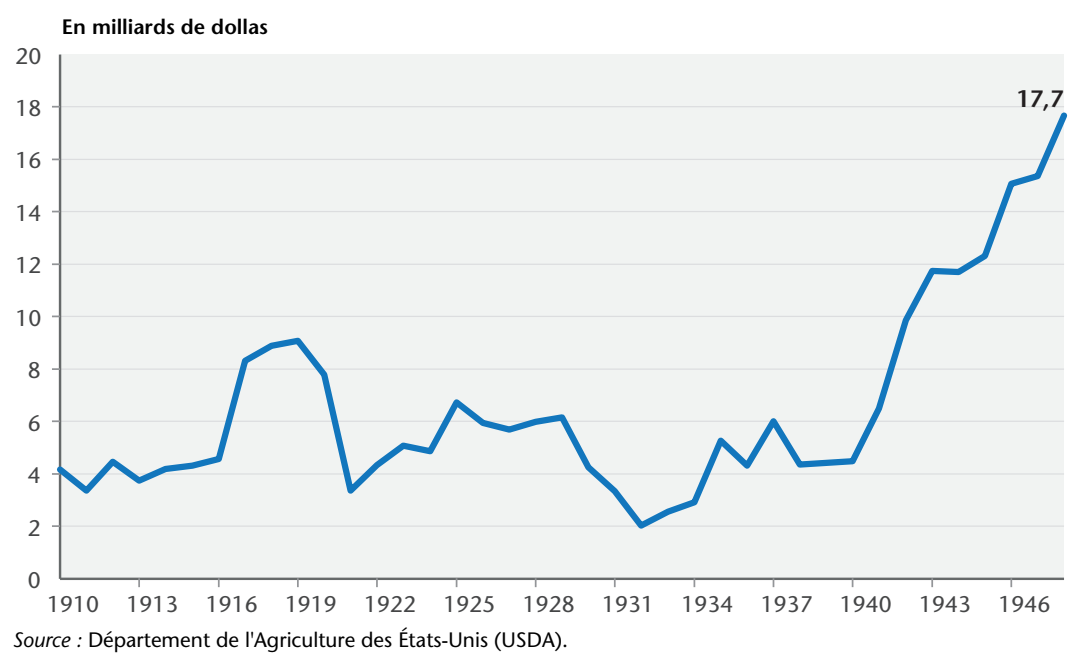

Céréales, viandes, vins..., la France a globalement contenu le risque de la famine durant le conflit grâce à l'intensité des échanges commerciaux de produits agricoles et alimentaires, lesquels se sont prolongés au-delà de la guerre. De 1914 à 1919, ces importations massives contribuent ainsi à une dégradation des comptes extérieurs du pays. D'excédentaire avant 1914, la balance commerciale agroalimentaire devient lourdement et chroniquement déficitaire jusqu'en 1919. Les années de guerre ont donc bel et bien bouleversé la structure du commerce extérieur français. On peut aller jusqu'à dire que, avec les importations de produits agricoles et alimentaires, c'est la hiérarchie des nations qui fournissent à la France ces denrées qui est bouleversée au profit des États-Unis (Becuwe et Blancheton, 2017). S'en est suivi à l'époque un rude débat autour de la liquidation du stock d'or de la France, pour honorer ses achats de denrées alimentaires (AugéLaribé, 1950).

Mobilisation des femmes, recours à la main-d'œuvre étrangère, ouverture des frontières commerciales, la guerre fait subir un véritable choc à son secteur agricole, même si l'idéal social de la $\mathrm{III}^{\mathrm{e}}$ République qu'il incarne encore, semble résister. Dans ce panorama des actions adoptées pendant la guerre, il manque toutefois une mesure phare qui, 
bien souvent, constitue un emblème de toute politique agricole : des prix garantis pour les producteurs. Vu d'Angleterre, qui a appliqué la garantie de prix en 1917, le refus de la France apparaît incompréhensible, dans la mesure où un prix garanti offre l'opportunité de voir la production augmenter. Mais par crainte d'un renforcement des pressions inflationnistes sur les prix alimentaires, les différents gouvernements ont renoncé à mettre en application ce principe du prix garanti. Les prix des céréales se sont de ce fait toujours situés en deçà des prix des produits importés.

La fin de la guerre approchant, sonne I'heure des bilans. Et ils sont sévères, les voix des parlementaires se mêlant à celles des économistes pour dénoncer l'inaction des gouvernements successifs, le recours excessif aux bonnes paroles et aux affirmations idéalisant la capacité de la France à se nourrir, plutôt qu'à l'action. Tout au plus est-il admis que le pays n'a pas sombré dans la pénurie, s'est «tiré d'affaire » comme I'indique Michel Augé-Laribé (page 359) ? $^{3}$ Tiré d'affaire en tombant dans une situation de dépendance vis-à-vis de l'extérieur, ce qui, à bien y regarder, ne concerne pas seulement la France. L'Allemagne est dans une situation bien pire, ainsi que d'autres pays européens, qui verront cette dépendance perdurer jusqu'à la veille de la Seconde Guerre mondiale (Asselain et Blancheton, 2000).

Partant de ce constat acerbe, comment la France a-t-elle reconstruit son appareil de production agricole à l'issue des combats et après la signature du Traité de Versailles? Quelle a été sa politique, quels ont été ses engagements pour redresser l'agriculture et faire en sorte qu'elle contribue au rétablissement de l'économie dans son ensemble?

\section{Ne rien changer, ou se doter d'un nouveau paradigme productif après la guerre?}

Dans son ouvrage dédié aux Conséquences économiques de la paix, John Maynard Keynes exprime son pessimisme quant à la situation de l'Europe à l'issue de la guerre. Selon lui, le Traité de Versailles ne contient que peu, voire aucune disposition destinée à rétablir l'économie de l'Europe, la priorité ayant été accordée à des motifs politiques visant à contrôler l'appareil économique de l'ennemi vaincu.

3. Dans son livre de 1950, Augé-Laribé souligne aussi l'état déplorable et les insuffisances de I'appareil statistique français sur le secteur agricole, ce qui a pu nuire aux responsables politiques en matière de prises de décisions. 
Keynes dresse en effet un constat très sévère sur l'état réel des économies d'Europe, au point de considérer que «l'Europe ne peut se suffire à elle-même. En particulier, elle n'a pas de quoi se nourrir (...) le danger qui nous menace est par conséquent la chute des conditions de vie des peuples européens jusqu'à un point qui, pour certains, sera la famine véritable (...) contre un pareil danger, nous devons unir toutes nos ressources, tout notre courage, tout notre idéalisme « (page 116). Plus loin est ajouté : «Quel est donc notre tableau de l'Europe ? Nous voyons une population rurale capable de vivre de ses produits agricoles sans pouvoir rien envoyer à la ville, et ne pouvant plus, comme jadis (par suite du manque de matériaux importés ou de produits négociables des villes), échanger de la nourriture contre d'autres marchandises » (page 118).

Ce diagnostic économique établi par l'auteur des Conséquences économiques de la paix est fort intéressant. Outre qu'il se penche sur la situation alimentaire de l'Europe, qu'il juge déplorable, sous-entendant fortement qu'il est indispensable de restaurer et surtout de développer la production agricole afin d'échapper aux risques de famines, Keynes prend pour illustration le cas de plusieurs pays dont celui de la France en reliant les dommages matériels réalisés durant les combats, et leurs conséquences sur l'état de l'agriculture française. II indique que : « un voyage à travers les régions dévastées de la France est plus impressionnant pour les yeux et l'imagination qu'il n'est possible de le dire. Durant I'hiver de 1918-1919, avant que la nature ait dissimulé la scène sous son manteau, l'horreur et la désolation apparaissaient aux yeux avec une grandeur effroyable. La destruction était complète. Sur des kilomètres et des kilomètres rien ne subsistait. Nul bâtiment ne pouvait être habité, nul champ ne pouvait être labouré. Une région dévastée était exactement pareille à une autre - un tas de gravats, un marécage de trous d'obus, de fils de fer tout embrouillés. Et cette similitude était impressionnante. La quantité de travail qui semblait nécessaire pour restaurer de telles régions semblait incalculable. Et le voyageur qui revenait de là-bas ne trouvait pas de sommes de milliards de livres capables d'exprimer la destruction gravée ainsi dans son esprit. Quelques gouvernements, pour diverses raisons, n'ont pas craint d'exploiter de telles impressions » (page 65). Ce diagnostic est partagé par Michel Augé-Laribé, qui explique dans son ouvrage consacré aux politiques agricoles de la France, que « toute l'agriculture, sur le territoire entier, pouvait être considérée comme sinistrée. Des terres abandonnées, mal entretenues pendant des années sont complétement à remettre en 
état », auteur qui ensuite indique que « l'agriculture était condamnée à faire dans toutes les branches de grands, rapides et ensuite continuels progrès, aboutissant à la réduction des prix de revient et à une situation satisfaisante pour les cultivateurs de toutes classes» (page 385). De quoi secouer les gouvernements afin qu'ils adoptent des mesures drastiques pour relever le défi de la croissance de la production agricole nationale. Et pourtant, le message de Keynes, la situation réelle des marchés agricoles, n'empêche nullement les occasions manquées de s'installer dans la durée.

La victoire militaire de la France s'inscrit dans ce contexte de défaite économique. Sur le plan agricole et alimentaire, elle a certes échappé au pire, grâce à des importations qui ont permis l'approvisionnement de la population civile et des soldats au front. Dès le lendemain de la guerre, gouvernement et Parlement, relayés par les organisations professionnelles agricoles, se lancent dans de vastes débats consacrés à la politique agraire à mener, aux moyens de restaurer la croissance de la production afin de nourrir une population ayant traversé quatre années de guerre, de souffrances, $d^{\prime}$ incertitudes. En matière agricole, la période qui s'ouvre après la fin des hostilités peut être vue comme celle des occasions manquées. Plus de quinze années vont passer avant de voir la France se doter d'une politique agricole offensive.

\subsection{Produire, mais selon quel modèle agricole?}

La guerre terminée, les hommes rentrent dans leurs fermes, du moins ceux qui seront restés valides. Le pays a récupéré l'Alsace et la Lorraine, et donc des hectares de terre cultivables. La capacité de produire est globalement restaurée. En 1925, la production agricole nationale retrouve son niveau d'avant-guerre avec ce surcroît de surfaces cultivables. L'accroissement annuel moyen de la production atteint, voire dépasse, les $2 \%$. Ce bon résultat ne conduit cependant pas le pays sur le chemin de l'autosuffisance alimentaire. La dépendance extérieure de la France durant l'entre-deux guerres ne s'accentue certes pas, mais ne se réduit pas pour autant (Toutain, 1992, 1993). Sur la période 1920-1938, c'est-à-dire jusqu'à la veille de la Seconde Guerre mondiale, la part de la production métropolitaine dans la consommation alimentaire des Français n'atteint pas les $100 \%$, à l'exception du beurre et des fromages. La production céréalière, y compris le froment, ne retrouve pas son niveau moyen d'avant 1914. II en découle que, de 1920 à 1938, les importations de céréales 
demeurent élevées, constituant même le cinquième poste des achats français à l'étranger ${ }^{4}$. En production viticole, malgré son redressement, les importations restent soutenues, puisque ce secteur occupe le troisième rang, devant les céréales. C'est pourquoi les approvisionnements en vins en provenance d'Algérie complètent ceux issus de transactions commerciales avec l'Allemagne. Quant aux produits oléagineux, la France renonce à les produire et se fournit quasi-intégralement à l'étranger, notamment en Afrique sub-saharienne qui exporte son arachide. La part des importations en provenance des colonies françaises s'accroît après la Première Guerre mondiale.

Comment restaurer la production agricole apparaît alors presque comme une question secondaire. Car, pour ainsi dire, plusieurs facteurs $s^{\prime}$ entremêlent pour mettre au jour la véritable question que se posent les responsables politiques et les professionnels, qui sont rejoints par les économistes de l'époque : quel est le modèle agraire à adopter pour la France après le conflit? Les effets démographiques de la guerre, la diminution de $30 \%$ des effectifs d'agriculteurs entre 1890 et 1920 en raison d'un exode rural engendré par l'urbanisation et l'industrialisation du pays, la sous-productivité du secteur, du fait de dépenses d'investissements insuffisants, une superficie moyenne d'exploitation agricole trop basse ( 8,3 hectares à la veille de la Grande Dépression), I'usage restreint des intrants chimiques (5 à $7 \mathrm{~kg}$ d'azote par hectare cultivé, contre 20 en Allemagne), posent pleinement la question du devenir d'une agriculture nationale, du changement de paradigme à la fois productif, organisationnel et institutionnel.

Une telle perspective, un tel impératif de reconstitution de l'appareil de production agricole font l'objet de débat au sein du gouvernement, entre ce dernier et les parlementaires, et entre les organisations professionnelles agricoles (Boussard, 1988). Les débats d'avant-guerre, lors de la crise agricole, évoqués plus haut dans cet article, resurgissent, avec sans doute davantage d'acuité. La ligne de démarcation se situe entre les modernistes, favorables à l'agrandissement de la taille des exploitations, aux investissements productifs, aux intrants chimiques, et les conservateurs agrariens, ces derniers entendant préserver la primauté de la cellule familiale en agriculture, ambition inséparable de la question de la propriété de la terre. En ce sens, ces controverses s'inscrivent dans

4. Il est par conséquent difficile de s'extirper de ce que Kevin $\mathrm{O}^{\prime}$ Rourke avait qualifié « d'invasion de grains en Europe », (O'Rourke, 2012). 
la profondeur de la crise qui secoue les «sociétés impériales», la Première Guerre en ayant formé une dimension tragique (Charles, 2001). La crise agricole, les interrogations qui prennent forme avant, durant et après la guerre quant aux mutations que le secteur doit accomplir et accepter, s'encastrent dans une crise plus générale des sociétés européennes, qui bouleverse les structures sociales.

Sur le plan politique, la vision de l'agriculture ne change guère. Elle est appréhendée comme le socle de l'ordre social. À droite de l'échiquier politique, l'interventionnisme étatique tel qu'il a été pratiqué durant la guerre est perçu comme suspect, porteur du danger de voir $s^{\prime}$ installer le socialisme dans les campagnes françaises. En réalité, cette crainte est infondée, dans la mesure où les gouvernements n'ayant fait, durant les combats, et dans un contexte dunion sacrée, que gérer une situation d'urgence complexe, pour ne pas défaillir en matière d'approvisionnement alimentaire. L'avènement de la collectivisation de l'agriculture est d'autant plus fantasmé par la droite que la gauche ellemême n'envisage pas un seul instant de copier le processus qui s'est déroulé en Union soviétique. Son projet agricole consiste à défendre l'exploitation familiale (le langage de la gauche de gouvernement use plutôt de l'expression «exploitation paysanne»), de petite et de moyenne dimension, et dont la rémunération de la production permet au paysan de faire vivre sa famille. C'est pourquoi la régulation des marchés doit constituer pour eux le fil conducteur de la politique agricole. Une perspective qui se concrétisera lors du Front Populaire comme nous allons le voir infra.

Dans l'immédiat, l'ambition première des gouvernements est triple : préserver et consolider la propriété familiale, favoriser l'expansion de la production, améliorer le mode de faire-valoir indirect, et protéger les salariés agricoles (durée journalière du travail, accidents du travail..., autant de décisions qui conduisent les organisations agrariennes à manifester leur hostilité, car elles y voient les signes annonciateurs de l'implantation du socialisme en agriculture). On se limitera ici aux deux premiers aspects. Sur le premier objectif, il s'agit de promouvoir les transactions foncières, de faciliter les achats de terre afin de contenir l'exode rural et de préserver l'équilibre ville-campagne, la production agricole restant ainsi une activité économique du ressort de I'initiative privée. En dépit du manque de stabilité politique qui affecte le secteur agricole en retardant les prises de décisions, plusieurs actions sont menées pour accélérer l'accès à la propriété des paysans. 
La Loi du 27 novembre 1918 facilite les opérations de remembrement. Elle est suivie, en 1920, de la création de l'Office national autonome du Crédit agricole (rebaptisé en 1926 Caisse nationale du Crédit Agricole), l'État souhaitant organiser un système de prêts bancaires individuels, support pour les paysans d'une acquisition du capital foncier.

Cette dynamique foncière répond aux suggestions formulées par Pierre Caziot (1876-1953), ingénieur agronome, spécialiste du foncier, représentant actif du courant agrarien, futur ministre de l'Agriculture et des approvisionnements du régime de Vichy entre 1940 et 1942, qui voyait dans l'articulation propriété-crédits bancaires, le vecteur de la formation de domaines familiaux de 10 à 20 hectares. II émit également le souhait de voir se construire, sous l'impulsion et la responsabilité de l'État, une Caisse nationale de la propriété foncière, dotée d'un droit de préemption lors des actes de ventes d'hectares de terre, souhait préfigurant les missions des S.A.F.E.R (Société d'aménagement foncier et d'établissement rural), qui seront instaurées sous la Ve République (Barral, 1968). Le Ministre Victor Boret (1872-1952), à qui l'on doit l'expression "à chaque famille son exploitation", s'empare de ces suggestions dans un projet de loi qui, face à l'hostilité politique des conservateurs, arc-boutés sur cette crainte de voir l'État envahir et diriger un secteur agricole, ne verra pas le jour. Une première étape de la reconstruction de l'agriculture qui avorte, au moins partiellement, laissant en vigueur les principes de politique agricole de la seconde moitié du XIX ${ }^{\mathrm{e}}$ siècle.

L'optimisme engendré par la victoire sur l'Allemagne contribue à maintenir l'illusion d'une agriculture efficace, performante, capable d'assurer à la fois l'approvisionnement de la population et l'idéal de l'ordre social cher à la III République. Pour cela, outre la question de la propriété foncière, c'est bien dans l'accroissement de la production agricole que réside, pour les responsables politiques, le secret du redressement du pays. L'objectif de la remise en état de fonctionnement économique les exploitations agricoles abîmées ou détruites par la guerre, est rapidement affiché. Pour cela, le versement d'indemnités aux agriculteurs est programmé, financées par les réparations de guerre. Une orientation nouvelle se dessine, puisque, au regard de la nette dégradation de la balance commerciale agricole durant la guerre, il s'agit pour la France, non seulement de se rapprocher de l'autosuffisance, mais de produire davantage pour exporter des surplus, et collecter ainsi des devises. 
L'autre vecteur du redressement productif a trait à la nécessité de fournir aux agriculteurs des engrais. Or, le Traité de Versailles, par la rétrocession de l'Alsace et la Lorraine qu'il implique, ramène dans le giron de la France les mines de potasse alsaciennes, à laquelle s'ajoute la cession du procédé de fabrication de l'ammoniaque (selon le procédé allemand Haber, qui constitua l'un des grands sujets lors des débats parlementaires après la guerre, en phase avec la reconstruction d'une industrie chimique en France). II en découle que la création de I'Office national de l'Azote en 1924, localisé à Toulouse, s'appuiera sur ce procédé pour fabriquer des engrais azotés ${ }^{5}$. Dans le même temps, les pouvoirs publics, sous l'impulsion du ministre de l'Agriculture Henri Queuille (1884-1970), lance un vaste programme d'électrification des campagnes. Le manque de capitaux dont souffrent les agriculteurs pour répondre au défi de la production sera comblé par le Crédit agricole, dont on a souligné, supra, la naissance en 1920, mais seulement partiellement étant donné la prégnance de l'épargne sur l'investissement qui prévaut encore dans les campagnes, la notion de dépenses sur le moyen et le long terme restant étrangère aux esprits des agriculteurs (Gervais, Jollivet, Tavernier, op. cit.).

La politique agricole menée au lendemain des combats est contenue dans un document du ministère de l'Agriculture et du Ravitaillement, daté de 1919, et publié dans le Bulletin National $4^{\circ}$ S. 2967, et qui s'intitule Avant-projet d'un programme agricole tendant à l'intensification de la production et à la réforme des méthodes administratives. Au Parlement, cette même année 1919, les élus de la République traitent de ce projet d'intensification de la production, à leurs yeux facteur essentiel de la prospérité de la France.

Toutes ces mesures n'entraînent pourtant pas le redressement productif attendu. D'abord parce que le processus de remembrement est fort lent, le morcellement des unités de production entravant la diffusion de la mécanisation, jugée indispensable par certains agronomes (Dumont, 1936) ${ }^{6}$. Ensuite, parce que les tensions et les rivalités entre les organisations professionnelles demeurent vivaces, notamment entre ce que les historiens du monde rural ont nommé « la rue

5. Office National de l'Azote dont Georges Patard, successeur de Louis Lheure au poste de Directeur des Poudres au ministère de la Guerre, fut le premier Directeur général.

6. La figure de René Dumont méritait d'être rappelée dans le cadre de cet article. Sa proximité politique avec les courants abondancistes des années 1930, emmenés par Jacques Duboin, lui permet de faire l'apologie du rôle de la technique et des machines dans la croissance agricole, de la hausse du revenu et donc de l'amélioration du bien-être des agriculteurs (Dard, 2015). 
d'Athènes " (la Société des Agriculteurs de France), dont les représentants se réclament d'un certain conservatisme en agriculture, appuyé par leur conviction catholique, et « le boulevard Saint-Germain » (la Société d'encouragement à l'agriculture), qui abrite des acteurs affichant leur radical-socialisme et qui sont favorables au progrès et à l'évolution des structures agraires. L'unité agricole, malgré la volonté de créer une Confédération nationale des associations agricoles, reste un vœu pieu.

Le secteur souffre également d'un manque d'institutions publiques et privées capables de le guider sur la voie du progrès, de le doter d'une représentativité auprès des pouvoirs publics. II faudra attendre les années 1920 pour voir émerger des Confédérations générales de producteurs (betteraves, blé, fruits et légumes), pour que les Chambres d'agriculture, créées en 1924 dans les départements, soient véritablement opérationnelles en 1927 avec la formation de I'Assemblée Permanente des Présidents de Chambres d'Agriculture (APPCA puis APCA), laquelle ne sera d'ailleurs reconnue que par un Décret du 30 octobre 1935, et accompagnent les agriculteurs dans leur diversité, dans leur évolution au gré de la politique agricole nationale. Les agriculteurs vont ainsi peu à peu être représentés auprès de l'État et du législateur (Atrux, 2012).

À vouloir maintenir un ordre social fondé sur les structures agraires, l'État et les conservateurs agrariens entravent le processus de modernisation de l'agriculture française après la guerre. Suspecté de vouloir introduire le socialisme dans ce secteur, l'État adopte des mesures de politique agricole qui ne bouleversent pas les structures sociales du monde paysan. II s'agit d'un interventionnisme a minima, qui ne franchit pas le seuil de l'exploitation agricole, presque au coup par coup, sans véritable cohérence d'ensemble, et qui s'inscrit de surcroît dans une politique économique générale libérale, qui privilégie manifestement l'industrie. Une occasion manquée à la veille de la Grande Dépression qui va plonger toutes les agricultures des pays industrialisés dans un marasme profond.

\subsection{La crise des années 1930 et la réponse de l'État français}

La fin de la Première Guerre mondiale, l'avènement de la paix, ne rétablissent pas les conditions de la production, ni ne mettent fin aux crises agricoles, celles-ci se concrétisant notamment par des fluctuations chroniques de prix. La France des années 1920 se distingue par une crise du secteur laitier, frappé par une surproduction qui oblige les 
acteurs des filières à innover, à mettre sur le marché de nouveaux produits, en particulier en fromages ou de beurre. Les autorités nationales, mais aussi celles des pays voisins de la France, se préoccupent de l'évolution du prix du blé. Blé et lait sont emblématiques des limites sur lesquelles butent le redressement de l'agriculture française, à partir de 1928, et plus encore lorsque la Grande Dépression s'abat sur une partie du monde capitaliste. Ces crises, auxquelles s'ajoute celle du secteur viticole du fait de l'intensification de la concurrence internationale, n'occasionnent pas pour autant un renoncement aux pratiques libérales instaurées après la guerre, même si, comme cela a été dit plus haut, l'État légifère sur certains domaines comme le foncier. Mais le marché agricole lui, épargné par l'interventionnisme étatique, ne fait pas jusque-là l'objet d'une quelconque régulation.

Les signes annonciateurs de l'émergence d'une régulation des marchés agricoles se situent dans la tenue de la Commission internationale de l'agriculture (CIA), qui, depuis la fin du XIX ${ }^{e}$ siècle, par-delà les politiques agricoles nationales, plus ou moins prononcées, se réunit pour que des échanges entre les acteurs des professions agricoles s'organisent et débouchent sur des orientations légitimant l'organisation des marchés. Après une interruption pendant la guerre, la Commission est reconnue comme institution agricole internationale et reprend ses travaux en 1927, dans le sillage de la Conférence économique internationale qui se déroule sous l'égide de la Société des Nations (SDN). La particularité de ces échanges est que les homologues allemands en sont exclus, illustrant une profonde rupture politique entre les nations, rupture qui absorbe le secteur agricole (Graevenitz, 2012). La problématique de la régulation des marchés agricoles n'est, après la guerre, pas séparable de la politique étrangère. Outre les réparations, l'Allemagne perd sa légitimité politique dans les instances internationales.

La reconnaissance de la CIA comme institution professionnelle agricole internationale constitue en quelque sorte une étape importante, dans la mesure où elle permet à l'agriculture européenne, à ses acteurs que sont les paysans, d'enclencher des réflexions sur l'état des marchés agricoles et sur leur régulation, sur la concurrence et la nécessité du protectionnisme, aboutissant à adresser aux gouvernements des demandes allant dans le sens de l'organisation des marchés, avec, en particulier, cette suggestion de créer des offices nationaux, peuvent être vus comme les vecteurs d'actions menées à l'échelle internationale pour équilibrer les marchés et discipliner la concurrence. En 1931, le secteur sucrier est à l'origine de la première tentative d'organisation 
des marchés et de mise en place d'offices nationaux, suivie en 1933, de la signature d'un Accord international sur le blé, issu de nombreuses discussions entre les experts agricoles pour promouvoir des conférences intergouvernementales sur la question des marchés, dans le but d'organiser celui du blé. Cet accord du 21 août 1933 est obtenu juste dans la foulée des résultats de la Conférence monétaire et économique qui s'est tenue à Londres. La finalité de l'Accord intergouvernemental sur le blé est de procéder d'une part à une augmentation du prix du blé, de façon à ce qu'il soit rémunérateur pour les producteurs - après l'effondrement du prix durant la crise de 1929-1930 - et, d'autre part, d'élaborer des dispositifs d'écoulement des stocks mondiaux. L'objectif de l'équilibre des marchés peut également être atteint par décision de réduire volontairement l'offre mondiale de blé par les principaux pays producteurs, en taxant par exemple les exportations ou en incitant les agriculteurs à réduire les surfaces emblavées (Argentine, États-Unis, Canada, France, URSS, Roumanie...). Étroitement relié à la Conférence monétaire et économique de Londres, cet accord sur le blé entre en résonance avec les travaux de nombreux économistes sur la question du fonctionnement des marchés, que ce soit des économistes américains, avec notamment Mordecai Ezékiel et son théorème du cobweb, ou britanniques, avec les analyses de Keynes sur la politique de stockage public des produits agricoles (Keynes, 1938).

L'agriculture française est particulièrement concernée par cette problématique de l'organisation des marchés. En effet, entre 1928 et 1932, le prix du blé s'effondre consécutivement à une surproduction et sous le coup d'importations qui, comme dans les années précédentes, restent importantes du fait des prix pratiqués par les principaux concurrents de la France. La crise mondiale submerge l'économie française à partir de 1931, amplifiant la baisse des prix agricoles. L'organisation des marchés étant encore très embryonnaire, des manifestations d'agriculteurs sont organisées, avec leur lot de violence, le point culminant se trouvant dans le meeting de l'été 1932, à la salle Wagram à Paris, où plus de dix mille agriculteurs expriment leur colère, obligeant le gouvernement à proposer, par la loi du 10 juillet 1933, un redressement des prix, mesure entravée par le maintien de la politique de libre-échange, au point que succède à cette loi, en décembre 1934, une autre loi rétablissant pleinement une politique libérale pour le secteur agricole (Lynch, 2019). Dans la continuité de l'après-guerre, une nouvelle occasion manquée. Car dans le même temps, les États-Unis, sous I'Administration Roosevelt, pour contenir la dislocation de l'agriculture 
nationale occasionnée par la crise, adopte un Agricultural Adjusment Act dès 1933, dont la finalité est de redresser les prix, de contrôler les volumes produits, y compris en recourant à la destruction des excédents, et, surtout, d'apporter une aide alimentaire aux Américains qui, avec la crise du capitalisme, connaissent une certaine paupérisation.

L'accession au pouvoir du Front populaire en 1936 constitue un tournant et dessine une nouvelle orientation de la politique agricole nationale. Loin des fantasmes diffusés par les forces politiques libérales, selon lesquels le Front populaire aurait préparé la collectivisation de l'agriculture - la propriété privée des outils de production ne fait à aucun moment l'objet d'une quelconque menace - il est au contraire question d'adopter des mesures d'urgence pour préserver le secteur agricole. La création de l'Office national interprofessionnel du blé (ONIB), en août 1936, symbolise à elle toute seule le tournant opéré par l'État en matière de politique agricole. Placé sous la double tutelle des ministères de l'Agriculture et des Finances, I'ONIB est doté du monopole des flux commerciaux de blé et doit procéder à la fixation des prix ainsi qu'à la séquence temporelle des achats de l'État auprès des producteurs. L'une des conséquences notables de la formation de I'ONIB se situe évidemment dans la nette remontée du prix du blé, puisque la hausse est de $125 \%$ entre 1935 et 1937 . Les voix hostiles à toute tentation d'organiser les marchés agricoles se raréfient, sombrant dans le silence par la suite puisque le régime de Vichy, en dépit du retour des agrariens conservateurs au pouvoir, ne reviendra pas sur cette institution, élargissant même ses prérogatives à l'ensemble des céréales (Gervais, Jollivet, Tavernier, op. cit.).

La crise agricole internationale, qui touche plus spécifiquement un produit comme le blé, conduit à un enchaînement de dispositifs étatiques pour stabiliser les marchés. L'organisation de ces marchés agricoles constituent une priorité qui semble partagée par de nombreux acteurs, mais qui s'est accompagnée de plusieurs contraintes (budgétaires, localisation géographique de l'Office du blé...). On ne saurait pour autant se limiter à cette dimension organisation des marchés. La longévité de la crise viticole en France, qui démarre en 1907, se caractérise notamment par le souhait de protéger des fraudes les vins produits sur le sol français. C'est pourquoi l'objectif de réprimer les fraudes, et de doter le secteur viti-vinicole d'un dispositif d'Appellations d'origine pour préserver les intérêts économiques de la profession lors de négociations commerciales multilatérales, se traduit par la création (Décret-Loi), en 1935, du Comité National des Appellations d'Origine 
des vins et eaux-de-vie, ancêtre de I'Institut National des Appellations $d^{\prime}$ Origine (INAO, créé en 1947) (Wolikow et Humbert, 2015) ${ }^{7}$.

Aux occasions manquées de l'après-Première Guerre mondiale, la crise des années 1930 constitue un nouveau choc qui conduit la gauche au pouvoir à opérer un revirement radical. Outre l'urgence avec laquelle il fallait répondre à la crise, et, en cela, il y a un point de convergence avec la situation agricole américaine, à trois ou quatre années d'intervalle, le tournant, cette fois-ci mieux négocié, de l'agriculture française, place celle-ci sur la voie de la modernisation. Ce tournant contient en gestation les outils d'une politique agricole moderne, qui va se déployer après la Second Guerre mondiale, et faire de l'agriculture nationale l'une des plus puissantes du monde. Régulation et organisation des marchés, stimulation de la production dans la perspective d'atteindre l'autosuffisance, autant de moyens et d'objectifs qui annoncent des temps nouveaux, ceux de l'Europe agricole, de la Politique agricole commune.

\section{Conclusion}

Une fois signé et appliqué le Traité de Versailles, le processus de reconstruction de l'agriculture française s'est étalé sur près d'une décennie, évoluant au gré des orientations retenues par les gouvernements en matière de politique économique générale et agricole en particulier. Une évolution heurtée, que dénote in fine les rapports de forces politiques mais aussi professionnels qui ont, soit souhaité faire entrer ce secteur dans la modernité, soit voulu retarder ce processus afin de préserver les équilibres sociaux hérités du XIX ${ }^{e}$ siècle. Les occasions manquées qui ont caractérisé la période d'après-guerre, ont puissamment contribué à l'érosion de la place de la France sur les marchés internationaux. II faudra attendre le milieu des années 1930 pour que soit esquissée une première rupture, celle de l'organisation des marchés céréaliers, rupture qui en annonce d'autres, d'ampleur bien plus élevée après la Seconde Guerre mondiale.

La période traitée dans cet article a, pour ainsi dire, une certaine résonance avec la période actuelle, le contexte étant évidemment bien

7. La formation du CNAO doit beaucoup à l'engagement, sur plusieurs décennies, de Joseph Capus (1867-1947). Agronome de formation, il fut ministre de l'Agriculture en 1924, puis député et sénateur entre 1919 et 1940 . C'est en mars 1935 qu'il proposa au Sénat un Projet de loi sur la protection des Appellations d'Origine. 
différent. L'agriculture française se cherche un nouveau paradigme productif, dresse l'amer constat de l'érosion de ses parts de marché, puisqu'elle s'est vue supplantée sur les marchés mondiaux par des puissances agricoles comme les Pays-Bas, l'Allemagne ou le Brésil. Au milieu du gué, le devenir des agriculteurs sera conditionné, comme par le passé, par le jeu des clivages politiques, par les fractures professionnelles opposant les anciennes générations ayant bénéficié des politiques agricoles actives d'après-1945, et les modernes, tournées vers les nouvelles technologies, la transition agro-écologique. Le poids des acteurs environnementalistes est à prendre en compte dans cette mutation, de même que les orientations fixées par l'Union européenne. Mais ce qui relie les deux périodes demeure dans la nécessité, voire dans l'impératif, de préserver une régulation des marchés agricoles. Ce sur quoi les acteurs ne convergent pas nécessairement.

\section{Références}

Anizan A.-L., 2014, «1914-1918, le gouvernement de guerre », Histoire@Politique.Politique, culture, société, numéro 22, janvier-avril, en ligne www.histoire-politique.fr (consulté le 17 août 2020).

Asselain J.-C., Blancheton B., 2000, «Les équilibres vitaux : la dépendance alimentaire de l'Europe », Revue économique, Vol. 51, n 2, p. 195-212.

Atrux M., 2012, « La naissance de l'ONIB et les réseaux professionnels agricoles : l'exemple des Chambres d'agriculture, 1936-1939 », in A. Chatriot, E. Leblanc, E. Lynch (éds.), Organiser les marchés agricoles. Le temps des fondateurs, Paris, éditions Armand Colin, p. 183-200.

Augé-Laribé M., 1950, La politique agricole de la France de 1880 à 1940, Paris, Presses Universitaires de France.

Barral P., 1968, Les agrariens de Méline à Pisani, Paris, Presses de la Fondation Nationale des Sciences Politiques.

Becuwe S., Blancheton B., 2017, "Le commerce extérieur français et la grande guerre : ruptures et continuités », Guerres mondiales et conflits contemporains, Vol. 2, n² 266, p. 79-98.

Bonin H., 2016, «La montée en puissance de la machine de guerre industrielle : vers une économie mixte (1914-1919)», Guerres mondiales et conflits contemporains, $\mathrm{n}^{\circ} 263, \mathrm{p} .123-146$.

Boussard I., 1988, "Les arguments économiques en faveur du retour à la terre dans le discours agrarien ", Economie rurale, numéros 184-185-186, mars-août, p. 75-84. 
Charle C., 2001, La Crise des sociétés impériales. Allemagne, France, GrandeBretagne, 1900-1940. Essai d'histoire sociale comparée, Paris, éditions du Seuil, coll. «UH ».

Dard O., 2015, "Sortir de la crise par la technique et par la science : les réponses de l'abondancisme de Jacques Duboin », L'Homme et la société, numéros 195-196, p. 127-146.

Dumont R., 1936, Misère ou prospérité paysannes, Paris, éditions Fustier, coll. «Dynamo ».

Gervais M., Jollivet M., Tavernier Y., 1977, La fin de la France paysanne. Depuis 1914, in G. Duby et A. Wallon (sous la dir.), Histoire de la France rurale, Tome 4, Paris, éditions du Seuil.

Graevenitz F.-G., 2012, «L'Europe comme modèle de l'Office du Blé ? Les origines et les conséquences nationales de l'organisation internationale des marchés agricoles (1927-1939), in A. Chatriot, E. Leblanc, E. Lynch (éds.), Organiser les marchés agricoles. Le temps des fondateurs, Paris, éditions Armand Colin, p. 53-86.

Keynes J.-M., 1920, Les conséquences économiques de la paix, Les classiques des sciences sociales, www.uqac.uquebec.ca/zone30/Classiques_des_sciences_sociales.index.html (consulté le 27 juillet 2020).

Keynes J.-M., 1938, « The Policy of Government Storage of Foodstuffs and Raw Materials », Economic Journal, 48, p. 458-469.

Kuisel R. F., 1984, Le capitalisme et l'État en France. Modernisation et dirigisme au $X X^{e}$ siècle, Paris, éditions Gallimard, Bibliothèque des Histoires.

Lhomme J., 1970, « La crise agricole à la fin du XIX ${ }^{\mathrm{e}}$ siècle. Essai d'interprétation économique et sociale », Revue économique, Vol. 21, n 4, p. 521-553.

Lynch E., 2019, Insurrections paysannes. De la terre à la rue. Usages de la violence au $X X^{e}$ siècle, Paris, éditions Vendémiaire.

O'Rourke K., 1997, «The European Grain Invasion. 1870-1913 », The Journal of Economic History, 57 (4), p. 775-801.

Pouch T., 2014, «La macroéconomie des années 1930 aux États-Unis : l'oubli de la politique agricole », Economie Appliquée, n 2, juin, p. 75-98.

Toutain J.-C., 1992, 1993, « La production agricole de la France de 1810 à 1990. Départements et régions. Croissance, productivité et structures, Economies et Sociétés, Cahiers de l'ISMEA, série AF, trois volumes, 11-12, 1-2 et 3-4.

Vigreux J., 2006, «Le passage d'un siècle à l'autre (1914-1929) : traumatismes et nouvelles donnes politiques », in J.-M. Moriceau et al. (éds.), Les campagnes dans les évolutions sociales et politiques en Europe. Des années 1830 à la fin des années 1920, Paris, éditions SEDES-CNED.

Wolikow S., Humbert F., 2015 (dirs.), Une histoire des vins et des produits d'AOC. L'INAO, de 1935 à nos jours, Dijon, éditions Universitaires de Dijon, coll. «Sociétés ».

Zolla D., 1904, Questions agricoles d'hier et d'aujourd'hui, Paris, éditions Armand Colin. 